\title{
Two parameter modified ratio estimators with two auxiliary variables for the estimation of finite population mean
}

\begin{abstract}
The present paper deals with some two parameter modified ratio estimators for the estimation of finite population means with known skewness and correlation coefficient on two auxiliary variables. It has been shown that the proposed modified ratio estimators perform better than the simple random sampling without replacement (SRSWOR) sample mean and some of the existing ratio estimators for certain natural populations available in the literature.
\end{abstract}

Keywords: mean squared error, natural populations, percentage relative efficiency, simple random sampling, ams subject classification: 62DJO5
Volume 7 Issue 6 - 2018

Jambulingam Subramani

Department of Statistics, Pondicherry University, India

Correspondence: Jambulingam Subramani, Department of Statistics, Pondicherry University, RV Nagar, Kalapet, Puducherry-605 014, India, Email drjsubramani@yahoo.co.in, drjsubramani@gmail.com

Received: October 22, 2018 | Published: December 14, 2018

\section{Introduction}

Let $\mathrm{Y}(\mathrm{X})$ be the study (auxiliary) variable taking values $Y_{i}\left(X_{i}\right)$ respectively on the unit $U_{i}, i=1,2, \ldots, N$ wherein $\mathrm{U}=\left\{\mathrm{U}_{1}, \mathrm{U}_{2}, \ldots, \mathrm{U}_{\mathrm{N}}\right\}$ be the finite population of size $\mathrm{N}$. The information on auxiliary variables are effectively used to improve the efficiency of the simple random sampling without replacement (SRSWOR) estimator of the population mean. As the results, ratio, product and regression estimators are widely utilized in many situations, see for example Cochran ${ }^{1}$ and Murthy. ${ }^{2}$ Modified ratio estimators are developed to achieve further improvements on the ratio estimator with known parameters of the auxiliary variable, which include Sisodia \& Dwivedi ${ }^{3}$ with known Co-efficient of Variation, Singh et., ${ }^{4}$ with known Kurtosis, Yan \& $\operatorname{Tian}^{5}$ with the known Skewness, Subramani and Kumarapandiyan ${ }^{6-9}$ with the known median and its linear combinations with the other known parameters. This paper deals with the two parameter modified ratio estimators with known correlation coefficient and skewness of two auxiliary variables $\mathrm{X}_{1}$ and $\mathrm{X}_{2}$ for estimating the population mean $\overline{\mathrm{Y}}$.

Several modified estimators have been proposed by linking together ratio, product and regression estimators in order to obtain more efficient estimators with two auxiliary variables. For a more detailed discussion about the estimators with two auxiliary variables one may refer to Abu-Dayyeh et al., ${ }^{10}$ Bandyopadhyay, ${ }^{11}$ Cochran, ${ }^{1}$ Kadilar \& Cingi, ${ }^{12,13}$ Khare et al., ${ }^{14}$ Murthy, ${ }^{2}$ Naik \& Gupta, ${ }^{15}$ Olkin I, ${ }^{16}$ Perri, ${ }^{17,18}$ Rao and Mudholkar, ${ }^{19}$ Raj, ${ }^{20}$ Sahoo \& Swain, ${ }^{21}$ Singh,${ }^{4}$ Singh, ${ }^{22,23}$ Singh \& Tailor, ${ }^{24}$ Srivenkataramana, ${ }^{25}$ Srivenkataramana \& Tracy, ${ }^{26}$ Tailor et al., ${ }^{27}$ Tracy et al., ${ }^{28}$ and the references cited there in.

The notations described below are used in this paper:

$\mathrm{N}$ - Population size

$\mathrm{n}$ - Sample size

$\mathrm{f}=\mathrm{n} / \mathrm{N}$, Sampling fraction

$\mathrm{Y}$ - Study variable

$\mathrm{X}_{1}$ and $\mathrm{X}_{2}-$ Auxiliary variables
$\overline{\mathrm{X}}_{1}, \overline{\mathrm{X}}_{2}, \overline{\mathrm{Y}}-$ Population means

$\overline{\mathrm{x}}_{1}, \overline{\mathrm{x}}_{2}, \overline{\mathrm{y}}-$ Sample means

$\hat{a}_{1}\left(X_{i}\right)-$ Coefficient of Skewness of auxiliary variable $X_{i}, i=1,2$

$\mathrm{R}_{1}=\frac{\overline{\mathrm{Y}}}{\overline{\mathrm{X}}_{1}}$ and $\mathrm{R}_{2}=\frac{\overline{\mathrm{Y}}}{\overline{\mathrm{X}}_{2}}$

$\mathrm{S}_{\mathrm{x}_{1}}, \mathrm{~S}_{\mathrm{x}_{2}}, \mathrm{~S}_{\mathrm{y}}-$ Population standard deviations

$\mathrm{S}_{\mathrm{x}_{1} \mathrm{y}}$ - Population covariance between $\mathrm{X}_{1}$ and $\mathrm{Y}$

$\mathrm{S}_{\mathrm{x}_{2} \mathrm{y}}$ - Population covariance between $\mathrm{X}_{2}$ and $\mathrm{Y}$

$\mathrm{S}_{\mathrm{x}_{1} \mathrm{x}_{2}}$ - Population covariance between $\mathrm{X}_{1}$ and $\mathrm{X}_{2}$

$\mathrm{C}_{\mathrm{x}}, \mathrm{C}_{\mathrm{x}_{1}}, \mathrm{C}_{\mathrm{x}_{2}}, \mathrm{C}_{\mathrm{y}}-$ Coefficient of variations

$\rho_{\mathrm{xy}}-$ Coefficient of correlation between $\mathrm{X}$ and $\mathrm{Y}$

$\rho_{\mathrm{yx}_{1}}-$ Coefficient of correlation between $\mathrm{Y}$ and $\mathrm{X}_{1}$

$\rho_{\mathrm{yx}_{2}}-$ Coefficient of correlation between $\mathrm{Y}$ and $\mathrm{X}_{2}$

$\rho_{\mathrm{x}_{1} \mathrm{x}_{2}}-$ Coefficient of correlation between $\mathrm{X}_{1}$ and $\mathrm{X}_{2}$

$\mathrm{B}_{1}=\frac{\mathrm{S}_{\mathrm{xy}}}{\mathrm{S}_{\mathrm{x}_{1}}^{2}}$, Regression coefficient of $\mathrm{Y}$ on $\mathrm{X}_{1}$

$\mathrm{B}_{2}=\frac{\mathrm{S}_{\mathrm{xy}}}{\mathrm{S}_{\mathrm{x}_{2}}^{2}}$, Regression coefficient of $\mathrm{Y}$ on $\mathrm{X}_{2}$

MSE(.) - Mean squared error of the estimator

$\tilde{\bar{Y}}_{i}-i^{\text {th }}$ Existing modified ratio estimator of $\bar{Y}$

In SRSWOR, the estimator of $\bar{Y}$ is $\bar{y}_{r}$ and its variance is

$\mathrm{V}\left(\overline{\mathrm{y}}_{\mathrm{r}}\right)=\frac{(1-\mathrm{f})}{\mathrm{n}} \mathrm{S}_{\mathrm{y}}^{2}$, where $\mathrm{S}_{\mathrm{y}}^{2}=\frac{1}{(\mathrm{~N}-1)} \sum_{\mathrm{i}=1}^{\mathrm{N}}\left(\mathrm{Y}_{\mathrm{i}}-\overline{\mathrm{Y}}\right)^{2}$

Cochran [3] introduced the classical ratio estimator for estimating the population mean $\overline{\mathrm{Y}}$ of the study variable $\mathrm{Y}$ using auxiliary 
variable $\mathrm{X}$ as given below:

$$
\widehat{\bar{Y}}_{\mathrm{R}}=\frac{\overline{\mathrm{y}}}{\overline{\mathrm{x}}} \overline{\mathrm{X}}=\hat{\mathrm{R}} \overline{\mathrm{X}} \text { where } \widehat{\mathrm{R}}=\frac{\overline{\mathrm{y}}}{\overline{\mathrm{x}}}=\frac{\mathrm{y}}{\mathrm{x}}
$$

The mean squared error of $\widehat{\bar{Y}}_{\mathrm{R}}$ to the first order of approximation is given below:

$$
\begin{aligned}
& \operatorname{MSE}\left(\widehat{\overline{\mathrm{Y}}}_{\mathrm{R}}\right)=\frac{(1-\mathrm{f})}{\mathrm{n}} \overline{\mathrm{Y}}^{2}\left(\mathrm{C}_{\mathrm{y}}^{2}+\mathrm{C}_{\mathrm{x}}^{2}-2\right. \\
& \qquad \operatorname{MSE}\left(\widehat{\overline{\mathrm{Y}}}_{1}\right)= \\
& \text { Using known correlation coefficient } \\
& \widehat{\overline{\mathrm{Y}}}_{2}=\overline{\mathrm{y}}\left(\frac{\overline{\mathrm{X}}_{1}+\rho_{\mathrm{x}_{1} \mathrm{x}_{2}}}{\overline{\mathrm{X}}_{1}+\rho_{\mathrm{x}_{1} \mathrm{x}_{2}}}\right)\left(\frac{\overline{\mathrm{X}}_{2}+\rho_{\mathrm{x}_{1 \times 2}}}{\overline{\bar{X}}_{2}+\rho_{\mathrm{x}_{1} \mathrm{x}_{2}}}\right)
\end{aligned}
$$

Singh [24] has suggested a ratio estimator with two auxiliary variables for estimating the population mean and is given below:

$$
\widehat{\overline{\mathrm{Y}}}_{1}=\overline{\mathrm{y}}\left(\frac{\overline{\mathrm{X}}_{1}}{\overline{\mathrm{x}}_{1}}\right)\left(\frac{\overline{\mathrm{X}}_{2}}{\overline{\mathrm{x}}_{2}}\right)
$$

The mean squared error of $\widehat{\bar{Y}}_{1}$ to the first order of approximation is given below:

The mean squared error of $\widehat{\bar{Y}}_{2}$ to the first order of approximation is given below:

$\operatorname{MSE}\left(\hat{\mathrm{Y}}_{2}\right)=\frac{(1-\mathrm{f})}{\mathrm{n}} \overline{\mathrm{Y}}^{2}\left[\mathrm{C}_{\mathrm{y}}^{2}+\mho_{1}^{*} \mathrm{C}_{\mathrm{x}_{1}}^{2}\left(\mho_{1}^{*}-2 \mathrm{~K}_{\mathrm{yx}_{1}}\right)+\mho_{2}^{*} \mathrm{C}_{\mathrm{x}_{2}}^{2}\left(\mho_{2}^{*}+2\left(\mathrm{~K}_{\mathrm{yx}_{2}}-\mho_{1}^{*} \mathrm{~K}_{\mathrm{x}_{1} \mathrm{x}_{2}}\right)\right)\right]$

where $\mathrm{K}_{\mathrm{yx} 1}=\rho_{\mathrm{yx} 1} \frac{\mathrm{C}_{\mathrm{y}}}{\mathrm{C}_{\mathrm{x}_{1}}}, \mathrm{~K}_{\mathrm{yx} 2}=\rho_{\mathrm{yx} 2} \frac{\mathrm{C}_{\mathrm{y}}}{\mathrm{C}_{\mathrm{x}_{2}}}, \mathrm{~K}_{\mathrm{x}_{1} \mathrm{x}_{2}}=\rho_{\mathrm{x}_{1} \mathrm{x}_{2}} \frac{\mathrm{C}_{\mathrm{x}_{1}}}{\mathrm{C}_{\mathrm{x}_{2}}}, \mho_{1}^{*}=\frac{\overline{\mathrm{X}}_{1}}{\overline{\mathrm{X}}_{1}+\rho_{\mathrm{x}_{1} \mathrm{x}_{2}}}$ and $\mho_{2}^{*}=\frac{\overline{\mathrm{X}}_{2}}{\overline{\mathrm{X}}_{2}+\rho_{\mathrm{x}_{1} \mathrm{x}_{2}}}$

Kadilar and Cingi [6] have proposed a new ratio estimator using two auxiliary variables and isgiven below:

$$
\widehat{\overline{\mathrm{Y}}}_{3}=\overline{\mathrm{y}}\left(\frac{\overline{\mathrm{X}}_{1}}{\overline{\mathrm{x}}_{1}}\right)^{1}\left(\frac{\overline{\mathrm{X}}_{2}}{\overline{\mathrm{x}}_{2}}\right)^{2}+\mathrm{b}_{1}\left(\overline{\mathrm{X}}_{1}-\overline{\mathrm{x}}_{1}\right)+\mathrm{b}_{2}\left(\overline{\mathrm{X}}_{2}-\overline{\mathrm{x}}_{2}\right)
$$

The mean squared error of $\widehat{\bar{Y}}_{3}$ to the first order of approximation is given below:

$$
\begin{aligned}
\operatorname{MSE}\left(\hat{\overline{\mathrm{Y}}}_{3}\right) \cong & \frac{(1-\mathrm{f})}{\mathrm{n}}\left[\mathrm{S}_{\mathrm{y}}^{2}+\left(\alpha_{1} \mathrm{R}_{1}+\mathrm{B}_{1}\right)^{2} \mathrm{~S}_{\mathrm{x}_{1}}^{2}+\left(\alpha_{2} \mathrm{R}_{2}+\mathrm{B}_{2}\right)^{2} \mathrm{~S}_{\mathrm{x}_{2}}^{2}-2\left(\alpha_{1} \mathrm{R}_{1}+\mathrm{B}_{1}\right) \mathrm{S}_{\mathrm{yx}}\right. \\
& \left.-2\left(\alpha_{2} \mathrm{R}_{2}+\mathrm{B}_{2}\right) \mathrm{S}_{\mathrm{yx}_{2}}+2\left(\alpha_{1} \mathrm{R}_{1}+\mathrm{B}_{1}\right)\left(\alpha_{2} \mathrm{R}_{2}+\mathrm{B}_{2}\right) \mathrm{S}_{\mathrm{x}_{1} \mathrm{x}_{2}}\right]
\end{aligned}
$$

Perri [13] has suggested some modified ratio cum product estimators using two auxiliary variables for estimating the population mean and are given below:

$$
\begin{aligned}
& \hat{\bar{Y}}_{4}=-\bar{y} \frac{\hat{\mathrm{t}}_{2}}{\hat{\mathrm{t}}_{1}} \frac{\overline{\mathrm{X}}_{1}}{\overline{\mathrm{X}}_{2}}, \hat{\bar{Y}}_{5}=-\overline{\mathrm{y}} \frac{\overline{\mathrm{X}}_{1}}{\hat{\mathrm{t}}_{1}} \frac{\overline{\mathrm{X}}_{2}}{\hat{\mathrm{t}}_{2}} \text { and } \hat{\overline{\mathrm{Y}}}_{6}=\overline{\mathrm{y}} \frac{\hat{\mathrm{t}}_{1}}{\hat{\mathrm{t}}_{2}} \frac{\overline{\mathrm{X}}_{2}}{\overline{\mathrm{X}}_{1}} \\
& \text { where } \hat{\mathrm{t}}_{1}=\overline{\mathrm{x}}_{1}+\alpha_{1}\left(\overline{\mathrm{X}}_{1}-\overline{\mathrm{x}}_{1}\right) \text { and } \hat{\mathrm{t}}_{2}=\overline{\mathrm{x}}_{2}+\alpha_{2}\left(\overline{\mathrm{X}}_{2}-\overline{\mathrm{x}}_{2}\right)
\end{aligned}
$$

The mean squared errors of $\widehat{\bar{Y}}_{4}, \widehat{\bar{Y}}_{5}$ and $\widehat{\bar{Y}}_{6}$ to the first order of approximation are given below:

$$
\begin{aligned}
& \operatorname{MSE}\left(\hat{\overline{\mathrm{Y}}}_{4}\right)=\frac{(1-\mathrm{f})}{\mathrm{n}}\left[\mathrm{S}_{\mathrm{y}}^{2}+\gamma_{\mathrm{x}_{1}}^{2}+\gamma_{\mathrm{x}_{2}}^{2}-2\left(\gamma_{\mathrm{yx}_{1}}-\gamma_{\mathrm{yx}_{2}}+\gamma_{\mathrm{x}_{1} \mathrm{x}_{2}}\right)\right] \\
& \operatorname{MSE}\left(\hat{\overline{\mathrm{Y}}}_{5}\right)=\frac{(1-\mathrm{f})}{\mathrm{n}}\left[\mathrm{S}_{\mathrm{y}}^{2}+\gamma_{\mathrm{x}_{1}}^{2}+\gamma_{\mathrm{x}_{2}}^{2}-2\left(\gamma_{\mathrm{yx}_{1}}+\gamma_{\mathrm{yx}_{2}}-\gamma_{\mathrm{x}_{1} \mathrm{x}_{2}}\right)\right]
\end{aligned}
$$

$$
\begin{aligned}
& \operatorname{MSE}\left(\widehat{\overline{\mathrm{Y}}}_{6}\right)=\frac{(1-\mathrm{f})}{\mathrm{n}}\left[\mathrm{S}_{\mathrm{y}}^{2}+\gamma_{\mathrm{x}_{1}}^{2}+\gamma_{\mathrm{x}_{2}}^{2}+2\left(\gamma_{\mathrm{yx}_{1}}-\gamma_{\mathrm{yx}_{2}}-\gamma_{\mathrm{x}_{1} \mathrm{x}_{2}}\right)\right](13) \\
& \text { where } \gamma_{\mathrm{x}_{1} \mathrm{x}_{2}}=\mathrm{R}_{1} \mathrm{R}_{2} \mathrm{~S}_{\mathrm{x}_{1} \mathrm{x}_{2}}\left(1-\alpha_{1}\right)\left(1-\alpha_{2}\right),_{\mathrm{x}_{1}}=\mathrm{R}_{1} \mathrm{~S}_{\mathrm{x}_{1}}\left(1-\alpha_{1}\right), \gamma_{\mathrm{x}_{2}}=\mathrm{R}_{2} \mathrm{~S}_{\mathrm{x}_{2}}\left(1-\alpha_{2}\right) \\
& \gamma_{\mathrm{yx}_{1}}=\mathrm{R}_{1} \mathrm{~S}_{\mathrm{yx}_{1}}\left(1-\alpha_{1}\right) \text { and } \gamma_{\mathrm{yx}_{2}}=\mathrm{R}_{2} \mathrm{~S}_{\mathrm{yx}_{2}}\left(1-\alpha_{2}\right)
\end{aligned}
$$

This paper is organized as follows: Section 2 introduces two parameter modified ratio estimators using the information of correlation coefficient and skewness of two auxiliary variables and the expressions for their bias and mean square error up to the first order of approximation have been derived. Section 3 is devoted to the analysis of the efficiencies of the proposed modified ratio estimators. In Section 4, an empirical analysis is carried out with some natural populations. Section 5 is ended with some concluding remarks.

\section{Proposed class of modified ratio estimators}

In this Section, two parameter modified ratio estimators with known correlation coefficient and skewness and their linear combinations of two auxiliary variables have been proposed and are given below:

$$
\begin{aligned}
& \widehat{\overline{\mathrm{Y}}}_{\mathrm{JS} 1}=-\overline{\mathrm{y}}\left(\frac{\alpha_{1} \overline{\mathrm{X}}_{1}+\alpha_{2} \overline{\mathrm{X}}_{2}+\rho_{\mathrm{x} 1 \mathrm{x}_{2}}}{\alpha_{1} \overline{\mathrm{x}}_{1}+\alpha_{2} \overline{\mathrm{x}}_{2}+\rho_{\mathrm{x} 1 \mathrm{x} 2_{2}}}\right) \\
& \widehat{\overline{\mathrm{Y}}}_{\mathrm{JS} 2}=\overline{\mathrm{y}}\left(\frac{\alpha_{1}\left(\overline{\mathrm{X}}_{1}+\beta_{1}\left(\mathrm{X}_{1}\right)\right)+{ }_{2}\left(\overline{\mathrm{X}}_{2}+\beta_{1}\left(\mathrm{X}_{2}\right)\right)}{\alpha_{1}\left(\overline{\mathrm{x}}_{1}+\beta_{1}\left(\mathrm{X}_{1}\right)\right)+{ }_{2}\left(\overline{\mathrm{x}}_{2}+\beta_{1}\left(\mathrm{X}_{2}\right)\right)}\right) \\
& \widehat{\overline{\mathrm{Y}}}_{\mathrm{JS} 3}=\overline{\mathrm{y}}\left(\frac{\alpha_{1}\left(\beta_{1}\left(\mathrm{X}_{1}\right) \overline{\mathrm{X}}_{1}+\rho_{\mathrm{x}_{1 \times 2}}\right)+\alpha_{2}\left(\beta_{1}\left(\mathrm{X}_{2}\right) \overline{\mathrm{X}}_{2}+\rho_{\mathrm{x}_{1 \times 2}}\right)}{\alpha_{1}\left(\beta_{1}\left(\mathrm{X}_{1}\right) \overline{\mathrm{x}}_{1}+\rho_{\mathrm{x}_{1} \mathrm{x}_{2}}\right)+\alpha_{2}\left(\beta_{1}\left(\mathrm{X}_{2}\right) \overline{\mathrm{x}}_{2}+\rho_{\mathrm{x}_{1} \mathrm{x}_{2}}\right)}\right) \\
& \widehat{\overline{\mathrm{Y}}}_{\mathrm{JS} 4}=\overline{\mathrm{y}}\left(\frac{\alpha_{1}\left(\rho_{\mathrm{x}_{1 \times} 2} \overline{\mathrm{X}}_{1}+\beta_{1}\left(\mathrm{X}_{1}\right)\right)+\alpha_{2}\left(\rho_{\mathrm{x}_{1 \times 2}} \overline{\mathrm{X}}_{2}+\beta_{1}\left(\mathrm{X}_{2}\right)\right)}{\alpha_{1}\left(\rho_{\mathrm{x}_{1} \times 2} \overline{\mathrm{x}}_{1}+\beta_{1}\left(\mathrm{X}_{1}\right)\right)+\alpha_{2}\left(\rho_{\mathrm{x}_{1} \mathrm{x} 2} \overline{\mathrm{x}}_{2}+\beta_{1}\left(\mathrm{X}_{2}\right)\right)}\right)
\end{aligned}
$$

In general the estimators proposed in (14) to (17) can be defined as given below:

$$
\widehat{\overline{\mathrm{Y}}}_{\mathrm{JSj}}=\overline{\mathrm{y}}\left(\frac{\alpha_{1}\left(\overline{\mathrm{X}}_{1}+\mathrm{T}_{1}\right)+\alpha_{2}\left(\overline{\mathrm{X}}_{2}+\mathrm{T}_{2}\right)}{\alpha_{1}\left(\overline{\mathrm{x}}_{1}+\mathrm{T}_{1}\right)+\alpha_{2}\left(\overline{\mathrm{x}}_{2}+\mathrm{T}_{2}\right)}\right), \mathrm{j}=1,2,3,4
$$

The mean squared errors of the proposed estimators are derived as given below:

Let $\mathrm{e}_{0}=\frac{\overline{\mathrm{y}}-\overline{\mathrm{Y}}}{\overline{\mathrm{Y}}}, \mathrm{e}_{1}=\frac{\overline{\mathrm{x}}_{1}-\overline{\mathrm{X}}_{1}}{\overline{\mathrm{X}}_{1}}$ and $\mathrm{e}_{2}=\frac{\overline{\mathrm{x}}_{2}-\overline{\mathrm{X}}_{2}}{\overline{\mathrm{X}}_{2}}$. Further we can write $\overline{\mathrm{y}}=\overline{\mathrm{Y}}\left(1+\mathrm{e}_{0}\right)$ 
$\overline{\mathrm{x}}_{1}=\overline{\mathrm{X}}_{1}\left(1+\mathrm{e}_{1}\right)$ and $\overline{\mathrm{x}}_{2}=\overline{\mathrm{X}}_{2}\left(1+\mathrm{e}_{2}\right)$ and from the definition of $\mathrm{e}_{0}$ and $\mathrm{e}_{1}$ we obtain:

$$
\begin{gathered}
\mathrm{E}\left[\mathrm{e}_{0}\right]=\mathrm{E}\left[\mathrm{e}_{1}\right]=0 \\
\mathrm{E}\left[\mathrm{e}_{0}^{2}\right]=\frac{(1-\mathrm{f})}{\mathrm{n}} \mathrm{C}_{\mathrm{y}}^{2}, \mathrm{E}\left[\mathrm{e}_{1}^{2}\right]=\frac{(1-\mathrm{f})}{\mathrm{n}} \mathrm{C}_{\mathrm{x}_{1}}^{2} \text { and } \mathrm{E}\left[\mathrm{e}_{2}^{2}\right]=\frac{1-\mathrm{f}}{\mathrm{n}} \mathrm{C}_{\mathrm{x}_{2}}^{2} \\
\mathrm{E}\left(\mathrm{e}_{0} \mathrm{e}_{1}\right)=\frac{(1-\mathrm{f})}{\mathrm{n}} \rho_{\mathrm{yx}_{1}} \mathrm{C}_{\mathrm{y}} \mathrm{C}_{\mathrm{x}_{1}}, \mathrm{E}\left(\mathrm{e}_{0} \mathrm{e}_{2}\right)=\frac{(1-\mathrm{f})}{\mathrm{n}} \rho_{\mathrm{yx}_{2}} \mathrm{C}_{\mathrm{y}} \mathrm{C}_{\mathrm{x}_{2}} \text { and } \mathrm{E}\left(\mathrm{e}_{1} \mathrm{e}_{2}\right)=\frac{(1-\mathrm{f})}{\mathrm{n}} \rho_{\mathrm{x}_{1} \mathrm{x}_{2}} \mathrm{C}_{\mathrm{x}_{1}} \mathrm{C}_{\mathrm{x}_{2}}
\end{gathered}
$$

The proposed estimators $\widehat{\bar{Y}}_{\mathrm{JSj}}$ can be written in terms of $\mathrm{e}_{0}, \mathrm{e}_{1}$ and $\mathrm{e}_{2}$ as given below:

$$
\begin{aligned}
& \widehat{\widehat{\mathrm{Y}}}_{\mathrm{JSj}}=\overline{\mathrm{Y}}\left(1+\mathrm{e}_{0}\right)\left(\frac{\alpha_{1}\left(\overline{\mathrm{X}}_{1}+\mathrm{T}_{1}\right)+\alpha_{2}\left(\overline{\mathrm{X}}_{2}+\mathrm{T}_{2}\right)}{\alpha_{1}\left(\overline{\mathrm{X}}_{1}\left(1+\mathrm{e}_{1}\right)+\mathrm{T}_{1}\right)+\alpha_{2}\left(\overline{\mathrm{X}}_{2}\left(1+\mathrm{e}_{2}\right)+\mathrm{T}_{2}\right)}\right) \\
& \Rightarrow \widehat{\overline{\mathrm{Y}}}_{\mathrm{JSj}}=\overline{\mathrm{Y}}\left(1+\mathrm{e}_{0}\right)\left(\frac{\alpha_{1} \overline{\mathrm{X}}_{1}+\alpha_{2} \overline{\mathrm{X}}_{2}+\alpha_{1} \mathrm{~T}_{1}+\alpha_{2} \mathrm{~T}_{2}}{\alpha_{1} \overline{\mathrm{X}}_{1}+\alpha_{2} \overline{\mathrm{X}}_{2}+\alpha_{1} \mathrm{~T}_{1}+\alpha_{2} \mathrm{~T}_{2}+\alpha_{1} \overline{\mathrm{X}}_{1} \mathrm{e}_{1}+\alpha_{2} \overline{\mathrm{X}}_{2} \mathrm{e}_{2}}\right) \\
& \Rightarrow \hat{\overline{\mathrm{Y}}}_{\mathrm{JSj}}=\overline{\mathrm{Y}}\left(1+\mathrm{e}_{0}\right)\left(\frac{1}{\left.1+\theta_{1}^{\prime} \mathrm{e}_{1}+\theta_{2}^{\prime} \mathrm{e}_{2}\right)}\right), \theta_{1}^{\prime}=\frac{\alpha_{1} \overline{\mathrm{X}}_{1}}{\alpha_{1}\left(\overline{\mathrm{X}}_{1}+\mathrm{T}_{1}\right)+\alpha_{2}\left(\overline{\mathrm{X}}_{2}+\mathrm{T}_{2}\right)}
\end{aligned}
$$

$$
\begin{aligned}
& \text { and } \theta_{2}^{\prime}=\frac{\alpha_{2} \overline{\mathrm{X}}_{2}}{\alpha_{1}\left(\overline{\mathrm{X}}_{1}+\mathrm{T}_{1}\right)+\alpha_{2}\left(\overline{\mathrm{X}}_{2}+\mathrm{T}_{2}\right)} \\
& \Rightarrow \widehat{\overline{\mathrm{Y}}}_{\mathrm{JSj}}=\overline{\mathrm{Y}}\left(1+\mathrm{e}_{0}\right)\left(1+\theta_{1}^{\prime} \mathrm{e}_{1}+\theta_{2}^{\prime} \mathrm{e}_{2}\right)^{-1} \\
& \Rightarrow \hat{\overline{\mathrm{Y}}}_{\mathrm{JSj}}=\overline{\mathrm{Y}}\left(1+\mathrm{e}_{0}\right)\left(1-\theta_{1}^{\prime} \mathrm{e}_{1}-\theta_{2}^{\prime} \mathrm{e}_{2}+\left(\theta_{1}^{\prime} \mathrm{e}_{1}+\theta_{2}^{\prime} \mathrm{e}_{2}\right)^{2}\right) \\
& \Rightarrow \widehat{\overline{\mathrm{Y}}}_{\mathrm{JSj}}=\overline{\mathrm{Y}}\left(1+\mathrm{e}_{0}\right)\left(1-\theta_{1}^{\prime} \mathrm{e}_{1}-\theta_{2}^{\prime} \mathrm{e}_{2}+\theta_{1}^{\prime 2} \mathrm{e}_{1}^{2}+\theta_{2}^{\prime 2} \mathrm{e}_{2}^{2}+2 \theta_{1}^{\prime} \mathrm{e}_{1} \theta_{2}^{\prime} \mathrm{e}_{2}\right)
\end{aligned}
$$

Neglecting the terms of higher order, we have

$$
\widehat{\overline{\mathrm{Y}}}_{\mathrm{JSj}}-\overline{\mathrm{Y}}=\overline{\mathrm{Y}} \mathrm{e}_{0}-\overline{\mathrm{Y}} \theta_{1}^{\prime} \mathrm{e}_{1}-\overline{\mathrm{Y}} \theta_{2}^{\prime} \mathrm{e}_{2}+\overline{\mathrm{Y}} \theta_{1}^{\prime} \mathrm{e}_{1} \theta_{2}^{\prime} \mathrm{e}_{2}-\overline{\mathrm{Y}} \theta_{1}^{\prime} \mathrm{e} \theta_{0} \mathrm{e}_{1}-\overline{\mathrm{Y}} \theta_{2}^{\prime} \mathrm{e} \theta_{0} \mathrm{e}_{2}
$$

Squaring and taking expectations on both sides, we have

$$
\begin{aligned}
& \operatorname{MSE}\left(\widehat{\overline{\mathrm{Y}}}_{\mathrm{JSj}}\right)=E\left(\widehat{\overline{\mathrm{Y}}}_{\mathrm{JSj}}-\overline{\mathrm{Y}}\right)^{2}=\overline{\mathrm{Y}}^{2} \mathrm{E}\left(\mathrm{e}_{0}-\theta_{1}^{\prime} \mathrm{e}_{1}-\theta_{2}^{\prime} \mathrm{e}_{2}\right)^{2} \\
\Rightarrow & \operatorname{MSE}\left(\hat{\overline{\mathrm{Y}}}_{\mathrm{JSj}}\right)=\overline{\mathrm{Y}}^{2} \mathrm{E}\left(\mathrm{e}_{0}^{2}+\theta_{1}^{\prime 2} \mathrm{e}_{1}^{2}+\theta_{2}^{\prime 2} \mathrm{e}_{2}^{2}-2 \theta_{1}^{\prime} \mathrm{e}_{0} \mathrm{e}_{1}-2 \theta_{2}^{\prime} \mathrm{e}_{0} \mathrm{e}_{2}+2 \theta_{1}^{\prime} \theta_{2}^{\prime} \mathrm{e}_{1} \mathrm{e}_{2}\right) \\
\Rightarrow & \operatorname{MSE}\left(\hat{\bar{Y}}_{\mathrm{JSj}}\right)=\overline{\mathrm{Y}}^{2}\left\{\mathrm{E}\left(\mathrm{e}_{0}^{2}\right)+\theta_{1}^{\prime 2} \mathrm{E}\left(\mathrm{e}_{1}^{2}\right)+\theta_{2}^{\prime 2} \mathrm{E}\left(\mathrm{e}_{2}^{2}\right)-2 \theta_{1}^{\prime} \mathrm{E}\left(\mathrm{e}_{0} \mathrm{e}_{1}\right)-2 \theta_{1}^{\prime} \mathrm{E}\left(\mathrm{e}_{0} \mathrm{e}_{2}\right)+2 \theta_{1}^{\prime} \theta_{2}^{\prime} \mathrm{E}\left(\mathrm{e}_{1} \mathrm{e}_{2}\right)\right\}
\end{aligned}
$$

$$
\operatorname{MSE}\left(\hat{\overline{\mathrm{Y}}}_{\mathrm{JSj}}\right)=\frac{(1-\mathrm{f})}{\mathrm{n}} \overline{\mathrm{Y}}^{2}\left\{\mathrm{C}_{\mathrm{y}}^{2}+\theta_{1}^{\prime 2} \mathrm{C}_{\mathrm{x}_{1}}^{2}+\theta_{2}^{\prime 2} \mathrm{C}_{\mathrm{x}_{2}}^{2}-2 \theta_{1}^{\prime} \rho_{\mathrm{yx}_{1}} \mathrm{C}_{\mathrm{y}} \mathrm{C}_{\mathrm{x}_{1}}-2 \theta_{2}^{\prime} \rho_{\mathrm{yx}_{2}} \mathrm{C}_{\mathrm{y}} \mathrm{C}_{\mathrm{x}_{2}}+2 \theta_{12}^{\prime \prime} \rho_{\mathrm{x}_{1} \mathrm{x}_{2}} \mathrm{C}_{\mathrm{x}_{1}} \mathrm{C}_{\mathrm{x}_{2}}\right\}
$$

\section{Efficiency comparisons}

In this Section the conditions for which the proposed estimators will have minimum mean squared error compared to SRSWOR sample mean and other existing estimators discussed in Section 2 for estimating the finite population mean have been derived algebraically.

From the expressions given in (18) and (1) the conditions for which the proposed estimator $\widehat{\overline{\mathrm{Y}}}_{\mathrm{JSj}}, \mathrm{j}=1,2,3,4$ is more efficient than the SRSWOR sample mean $\overline{\mathrm{y}}_{\mathrm{r}}$ have been derived and are given below:

$\operatorname{MSE}\left(\hat{\overline{\mathrm{Y}}}_{\mathrm{JSj}}\right) \leq \mathrm{V}\left(\overline{\mathrm{y}}_{\mathrm{r}}\right)$ if $\theta_{1}^{\prime 2} \mathrm{C}_{\mathrm{x}_{1}}^{2}+\theta_{2}^{\prime 2} \mathrm{C}_{\mathrm{x}_{2}}^{2} \leq 2\left(\theta_{1}^{\prime} \rho_{\mathrm{yx}_{1}} \mathrm{C}_{\mathrm{y}} \mathrm{C}_{\mathrm{x}_{1}}+\theta_{2}^{\prime} \rho_{\mathrm{yx}_{2}} \mathrm{C}_{\mathrm{y}} \mathrm{C}_{\mathrm{x}_{2}}-\theta_{1}^{\prime} \theta_{2}^{\prime} \rho_{\mathrm{x}_{1} \mathrm{x}_{2}} \mathrm{C}_{\mathrm{x}_{1}} \mathrm{C}_{\mathrm{x}_{2}}\right)$

From the expressions given in (18) and (5) the conditions for which the proposed estimator $\widehat{\bar{Y}}_{\mathrm{JSj}}, \mathrm{j}=1,2,3,4$ is more efficient than the existing ratio estimator $\widehat{\bar{Y}}_{1}$ have been derived and are given below:

$$
\begin{aligned}
& \operatorname{MSE}\left(\widehat{\overline{\mathrm{Y}}}_{\mathrm{JSj}}\right) \leq \operatorname{MSE}\left(\widehat{\overline{\mathrm{Y}}}_{1}\right) \text { if }\left(\theta_{1}^{\prime 2}-1\right) \mathrm{C}_{\mathrm{x}_{1}}^{2}+\left(\theta_{1}^{\prime 2}-1\right) \mathrm{C}_{\mathrm{x}_{2}}^{2} \\
& \leq 2\left\{\left(\theta_{1}^{\prime}-1\right) \rho_{\mathrm{yx}_{1}} \mathrm{C}_{\mathrm{y}} \mathrm{C}_{\mathrm{x}_{1}}+\left(\theta_{2}^{\prime}-1\right) \rho_{\mathrm{yx}_{2}} \mathrm{C}_{\mathrm{y}} \mathrm{C}_{\mathrm{x}_{2}}-\left(\theta_{1}^{\prime} \theta_{2}^{\prime}-1\right) \rho_{\mathrm{x}_{1} \mathrm{x}_{2}} \mathrm{C}_{\mathrm{x}_{1}} \mathrm{C}_{\mathrm{x}_{2}}\right\}
\end{aligned}
$$

From the expressions given in (18) and (7) the conditions for which the proposed estimator $\hat{\bar{Y}}_{\mathrm{JSj}}, \mathrm{j}=1,2,3,4$ is more efficient than the existing ratio estimator $\hat{\bar{Y}}_{2}$ have been derived and are given below:

$$
\begin{aligned}
& \operatorname{MSE}\left(\hat{\overline{\mathrm{Y}}}_{\mathrm{JSj}}\right) \leq \operatorname{MSE}\left(\hat{\overline{\mathrm{Y}}}_{2}\right) \text { if }\left(\theta_{1}^{\prime 2}-{ }_{1}^{* 2}\right) \mathrm{C}_{\mathrm{x}_{1}}^{2}+\left(\theta_{2}^{\prime 2}-{ }_{2}^{* 2}\right) \mathrm{C}_{\mathrm{x}_{2}}^{2} \\
& \leq 2\left\{\left(\theta_{1}^{\prime}-\mho_{1}^{*}\right) \rho_{\mathrm{yx}_{1}} \mathrm{C}_{\mathrm{y}} \mathrm{C}_{\mathrm{x}_{1}}+\left(\theta_{2}^{\prime}+\mho_{2}^{*}\right) \rho_{\mathrm{yx}_{2}} \mathrm{C}_{\mathrm{y}} \mathrm{C}_{\mathrm{x}_{2}}-\left(\theta_{1}^{\prime} \theta_{2}^{\prime}+\mho_{1}^{*} \widetilde{\mho}_{2}^{*}\right) \rho_{\mathrm{x}_{1} \mathrm{x}_{2}} \mathrm{C}_{\mathrm{x}_{1}} \mathrm{C}_{\mathrm{x}_{2}}\right\}
\end{aligned}
$$

From the expressions given in (18) and (9) the conditions for which the proposed estimator $\widehat{\overline{\mathrm{Y}}}_{\mathrm{JSj}}, \mathrm{j}=1,2,3,4$ is more efficient than the existing ratio estimator $\widehat{\bar{Y}}_{3}$ have been derived and are given below:

$$
\begin{aligned}
& \operatorname{MSE}\left(\hat{\overline{\mathrm{Y}}}_{\mathrm{JSj}}\right) \leq \operatorname{MSE}\left(\hat{\overline{\mathrm{Y}}}_{3}\right) \text { if } \alpha_{1}^{2}\left(\mathrm{R}_{\mathrm{js}}^{\prime}{ }^{2}-\mathrm{R}_{1}^{2}\right) \mathrm{S}_{\mathrm{x}_{1}}^{2}+\alpha_{2}^{2}\left(\mathrm{R}_{\mathrm{js}}^{\prime}{ }^{2}-\mathrm{R}_{2}^{2}\right) \mathrm{S}_{\mathrm{x}_{2}}^{2}+\mathrm{B}_{1} S_{\mathrm{yx}_{1}}+\mathrm{B}_{2} S_{\mathrm{yx}_{2}} \\
& \leq 2\left\{\mathrm{R}_{\mathrm{js}}^{\prime}\left(\alpha_{1} S_{\mathrm{yx}_{1}}+\alpha_{2} S_{\mathrm{yx}_{2}}\right)-\left[\alpha_{1} \alpha_{2} \mathrm{R}_{\mathrm{js}}^{\prime}{ }^{2}-\left(\alpha_{1} \mathrm{R}_{1}+\mathrm{B}_{1}\right)\left(\alpha_{2} \mathrm{R}_{2}+\mathrm{B}_{2}\right)\right] \mathrm{S}_{\mathrm{x}_{1} \mathrm{x}_{2}}\right\}
\end{aligned}
$$

From the expressions given in (18) and (11) the conditions for which the proposed estimator $\widehat{\overline{\mathrm{Y}}}_{\mathrm{JSj}} \mathrm{j}=1,2,3,4$ is more efficient than the existing ratio estimator $\widehat{\bar{Y}}_{4}$ have been derived and are given below:

$$
\begin{aligned}
& \operatorname{MSE}\left(\hat{\overline{\mathrm{Y}}}_{\mathrm{JSj}}\right) \leq \operatorname{MSE}\left(\hat{\overline{\mathrm{Y}}}_{4}\right) \text { if }\left[\alpha_{1}^{2} \mathrm{R}_{\mathrm{js}}^{\prime 2}-\left(1-\alpha_{1}\right)^{2} \mathrm{R}_{1}^{2}\right] \mathrm{S}_{\mathrm{x}_{1}}^{2}+\left[\alpha_{2}^{2} \mathrm{R}_{\mathrm{js}}^{\prime 2}-\left(1-\alpha_{2}\right)^{2} \mathrm{R}_{2}^{2}\right] \mathrm{S}_{\mathrm{x}_{2}}^{2} \\
& \leq 2\left\{S_{\mathrm{yx}}\left[\alpha_{1} \mathrm{R}_{\mathrm{js}}^{\prime}-\left(1-\alpha_{1}\right) \mathrm{R}_{1}\right]+S_{\mathrm{yx}_{2}}\left[\alpha_{2} \mathrm{R}_{\mathrm{js}}^{\prime}+\left(1-\alpha_{2}\right) \mathrm{R}_{2}\right]-\mathrm{S}_{\mathrm{x}_{1 \times 2}}\left[\alpha_{1} \alpha_{2} \mathrm{R}_{\mathrm{js}}^{2}+\mathrm{R}_{1} \mathrm{R}_{2}\left(1-\alpha_{1}\right)\left(1-\alpha_{2}\right)\right]\right\}
\end{aligned}
$$

From the expressions given in (18) and (12) the conditions for which the proposed estimator $\widehat{\overline{\mathrm{Y}}}_{\mathrm{JSj}}, \mathrm{j}=1,2,3,4$ is more efficient than the existing ratio estimator $\widehat{\bar{Y}}_{5}$ have been derived and are given below: 


$$
\begin{aligned}
& \operatorname{MSE}\left(\hat{\overline{\mathrm{Y}}}_{\mathrm{JSj}}\right) \leq \operatorname{MSE}\left(\hat{\overline{\mathrm{Y}}}_{5}\right) \text { if }\left[\alpha_{1}^{2} \mathrm{R}_{\mathrm{js}}^{\prime}{ }^{2}-\left(1-\alpha_{1}\right)^{2} \mathrm{R}_{1}^{2}\right] \mathrm{S}_{\mathrm{x}_{1}}^{2}+\left[\alpha_{2}^{2} \mathrm{R}_{\mathrm{js}}^{\prime}{ }^{2}-\left(1-\alpha_{2}\right)^{2} \mathrm{R}_{2}\right] \mathrm{S}_{\mathrm{x}_{2}} \\
& \leq 2\left\{S_{\mathrm{yx}_{1}}\left[\alpha_{1} \mathrm{R}_{\mathrm{js}}^{\prime}-\left(1-\alpha_{1}\right) \mathrm{R}_{1}\right]+S_{\mathrm{yx}_{2}}\left[\alpha_{2} \mathrm{R}_{\mathrm{js}}^{\prime}-\left(1-\alpha_{2}\right) \mathrm{R}_{2}\right]-\mathrm{S}_{\mathrm{x}_{1} \mathrm{x}_{2}}\left[\alpha_{1} \alpha_{2} \mathrm{R}_{\mathrm{js}}^{\prime}{ }^{2}-\mathrm{R}_{1} \mathrm{R}_{2}\left(1-\alpha_{1}\right)\left(1-\alpha_{2}\right)\right]\right\}
\end{aligned}
$$

From the expressions given in (18) and (13) the conditions for which the proposed estimator $\hat{\bar{Y}}_{\mathrm{JSj}} \mathrm{j}=1,2,3,4$ is more efficient than the existing ratio estimator $\widehat{\bar{Y}}_{6}$ have been derived and are given below:

$$
\begin{aligned}
& \operatorname{MSE}\left(\hat{\overline{\mathrm{Y}}}_{\mathrm{JSj}}\right) \leq \operatorname{MSE}\left(\hat{\overline{\mathrm{Y}}}_{6}\right) \text { if }\left[\alpha_{1}^{2} \mathrm{R}_{\mathrm{js}}^{\prime}{ }^{2}-\left(1-\alpha_{1}\right)^{2} \mathrm{R}_{1}^{2}\right] \mathrm{S}_{\mathrm{x}_{1}}^{2}+\left[\alpha_{2}^{2} \mathrm{R}_{\mathrm{js}}^{\prime 2}-\left(1--_{2}\right)^{2} \mathrm{R}_{2}^{2}\right] \mathrm{S}_{\mathrm{x}_{2}}^{2} \\
& \leq 2\left\{S_{\mathrm{yx}_{1}}\left[\alpha_{1} \mathrm{R}_{\mathrm{js}}^{\prime}+\left(1-\alpha_{1}\right) \mathrm{R}_{1}\right]+S_{\mathrm{yx}_{2}}\left[\alpha_{2} \mathrm{R}_{\mathrm{js}}^{\prime}-\left(1-\alpha_{2}\right) \mathrm{R}_{2}\right]-\mathrm{S}_{\mathrm{x}_{1} \mathrm{x}_{2}}\left[\alpha_{1} \alpha_{2} \mathrm{R}_{\mathrm{js}}^{\prime}{ }^{2}+\mathrm{R}_{1} \mathrm{R}_{2}\left(1-\alpha_{1}\right)\left(1-\alpha_{2}\right)\right]\right\}
\end{aligned}
$$

where $\mathrm{R}_{\mathrm{js}}^{\prime}=\frac{\overline{\mathrm{Y}}}{\alpha_{1}\left(\overline{\mathrm{X}}_{1}+\mathrm{T}_{1}\right)+{ }_{2}\left(\overline{\mathrm{X}}_{2+} \mathrm{T}_{2}\right)}$

\section{Numerical comparisons}

The performance of the proposed modified ratio estimators are assessed with that of the SRSWOR sample mean and the existing

modified ratio estimators for certain natural populations. In this connection, we have considered two natural populations for the assessment of the performance of the proposed estimators with that of the existing estimators. The population 1 is taken from Singh \& Chaudhary ${ }^{29}$ given in page 177 and population 2 is taken from taken from the Cingi \& Kadilar $^{30}$ given in page 117. The description of the study variable and auxiliary variable for the two populations are given below: (Table 1-4).

\begin{tabular}{|c|c|c|}
\hline Parameters & Population I & Population 2 \\
\hline $\mathrm{N}$ & 34 & 25 \\
\hline $\mathrm{n}$ & 20 & 10 \\
\hline$\overline{\mathrm{Y}}$ & 856.41 & 75.28 \\
\hline$\overline{\mathrm{X}}_{1}$ & 208.88 & 14.3 \\
\hline $\bar{X}_{2}$ & 199.44 & 6.82 \\
\hline$\rho_{\mathrm{yx}_{1}}$ & 0.45 & 0.99 \\
\hline$\rho_{\mathrm{yx} 2}$ & 0.45 & 0.89 \\
\hline$\rho_{\mathrm{x}_{1} \mathrm{x}_{2}}$ & 0.98 & 0.92 \\
\hline$\beta_{11}$ & 0.87 & 1.24 \\
\hline$\beta_{12}$ & 1.28 & 0.86 \\
\hline$\beta_{21}$ & 2.91 & 4.26 \\
\hline$\beta_{22}$ & 3.73 & 4.35 \\
\hline $\mathrm{S}_{\mathrm{y}}$ & 733.14 & 17.27 \\
\hline $\mathrm{C}_{\mathrm{y}}$ & 0.86 & 0.23 \\
\hline $\mathrm{S}_{\mathrm{x}_{1}}$ & $|50.5|$ & 3.17 \\
\hline $\mathrm{S}_{\mathrm{x}_{2}}$ & 150.22 & 1.53 \\
\hline $\mathrm{C}_{\mathrm{x}_{1}}$ & 0.72 & 0.22 \\
\hline $\mathrm{C}_{\mathrm{x}_{2}}$ & 0.75 & 0.22 \\
\hline
\end{tabular}

Table I Description of the study variable and auxiliary variable

\begin{tabular}{llll}
\hline Popl. No. & Study variable- $\mathbf{Y}$ & Auxiliary variable- $\mathbf{X}_{1}$ & Auxiliary variable- $\mathbf{X}_{2}$ \\
\hline I & Area under wheat in I974 & Area under wheat in I97I & Area under wheat in 1973 \\
2 & $\begin{array}{l}\text { The population mean of the } \\
\text { height of the fish }\end{array}$ & $\begin{array}{l}\text { The population mean of the } \\
\text { length of the head }\end{array}$ & $\begin{array}{l}\text { The population mean of the } \\
\text { length of the fin }\end{array}$ \\
\hline
\end{tabular}

Table 2 Parameters and constants of the populations 
Table 3 Variance/Mean squared error of the existing and proposed estimators for the Population I

\section{Existing estimators}

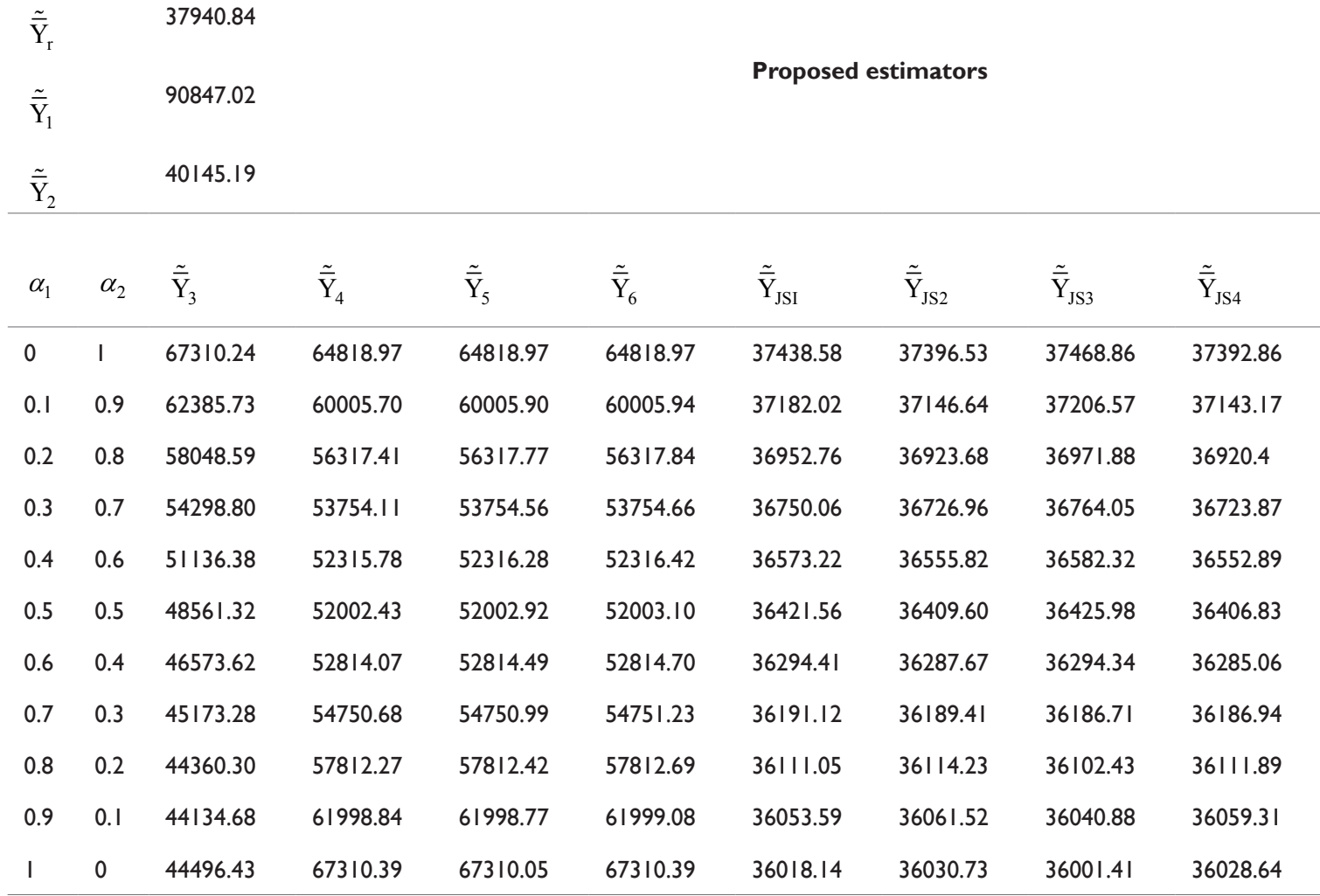

Table 4 Variance/Mean squared error of the existing and proposed estimators for the Population 2

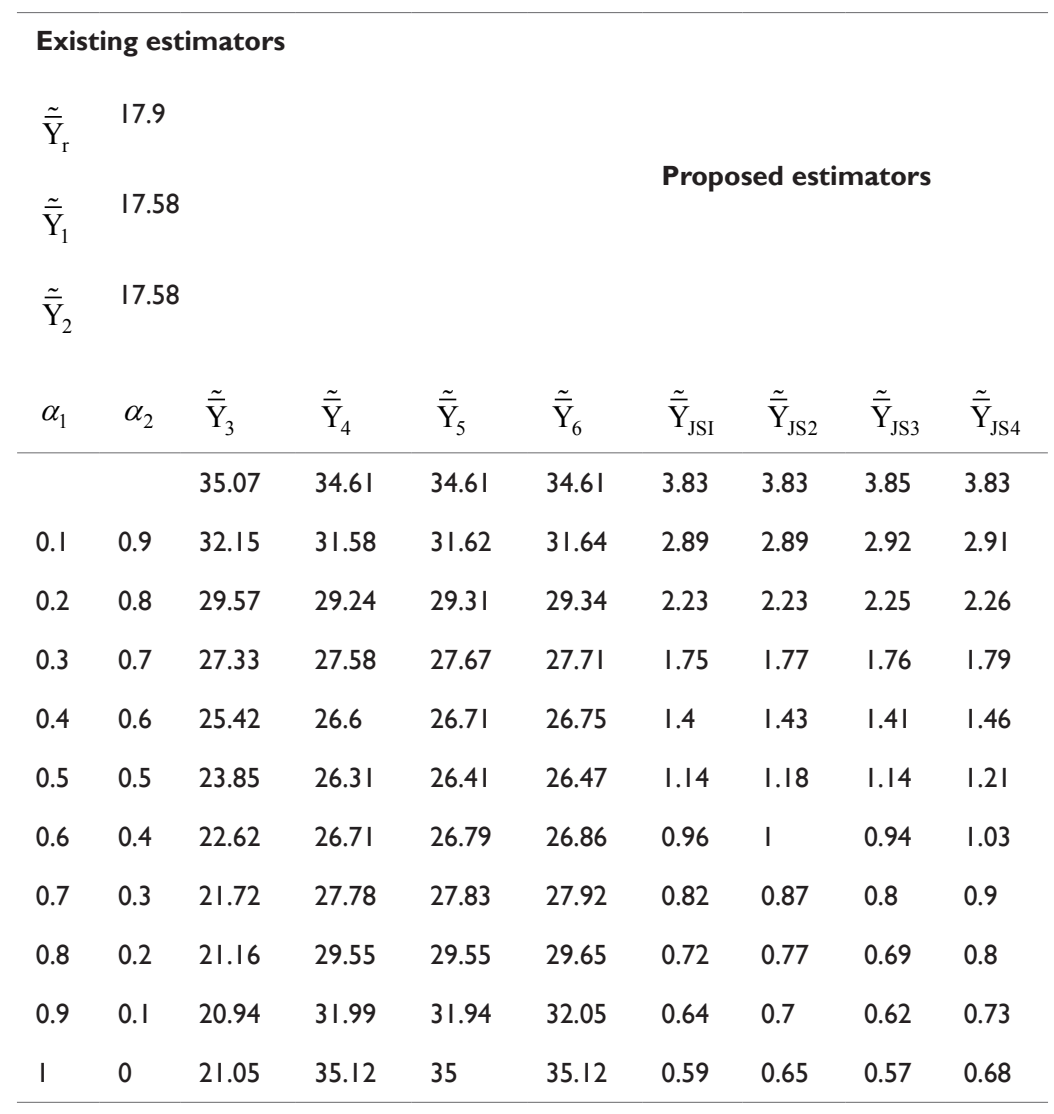

Citation: Subramani J.Two parameter modified ratio estimators with two auxiliary variables for the estimation of finite population mean. Biom Biostat Int J. 2018;7(6):559-568. DOI: 10.15406/bbij.2018.07.00259 
The population parameters and constants computed for the above two populations are given below:

From the values of Table 5-12, it is observed that the proposed modified ratio estimators perform better than SRSWOR sample mean and the existing modified ratio estimators. ${ }^{30-35}$

Table 5 PRE of the proposed estimator $\tilde{\bar{Y}}_{\text {JS1 }}$ for the Population I

\begin{tabular}{|c|c|c|c|c|c|c|c|c|}
\hline \multirow[b]{2}{*}{$\alpha_{1}$} & \multirow[b]{2}{*}{$\alpha_{2}$} & \multirow{2}{*}{$\begin{array}{l}\text { SRSWOR } \\
\bar{y}_{\mathbf{r}}\end{array}$} & \multicolumn{6}{|c|}{ Existing estimators } \\
\hline & & & $\tilde{\overline{\mathrm{Y}}}_{1}$ & $\tilde{\overline{\mathrm{Y}}}_{2}$ & $\tilde{\overline{\mathrm{Y}}}_{3}$ & $\tilde{\overline{\mathrm{Y}}}_{4}$ & $\tilde{\overline{\mathrm{Y}}}_{5}$ & $\tilde{\overline{\mathrm{Y}}}_{6}$ \\
\hline 0 & 1 & 101.34 & 242.66 & 107.23 & 179.79 & 173.13 & 173.13 & 173.13 \\
\hline 0.1 & 0.9 & 102.04 & 244.33 & 107.97 & 167.78 & 161.38 & 161.38 & 161.38 \\
\hline 0.2 & 0.8 & 102.67 & 245.85 & 108.64 & 157.09 & 152.4 & 152.4 & 152.4 \\
\hline 0.3 & 0.7 & 103.24 & 247.2 & 109.24 & 147.75 & 146.27 & 146.27 & 146.27 \\
\hline 0.4 & 0.6 & 103.74 & 248.4 & 109.77 & 139.82 & 143.04 & 143.05 & 143.05 \\
\hline 0.5 & 0.5 & 104.17 & 249.43 & 110.22 & 133.33 & 142.78 & 142.78 & 142.78 \\
\hline 0.6 & 0.4 & 104.54 & 250.31 & 110.61 & 128.32 & 145.52 & 145.52 & 145.52 \\
\hline 0.7 & 0.3 & 104.83 & 251.02 & 110.93 & 124.82 & 151.28 & 151.28 & 151.28 \\
\hline 0.8 & 0.2 & 105.07 & 251.58 & 111.17 & 122.84 & 160.1 & 160.1 & 160.1 \\
\hline 0.9 & 0.1 & 105.23 & 251.98 & 111.35 & $|22.4|$ & 171.96 & 171.96 & 171.96 \\
\hline 1 & 0 & 105.34 & 252.23 & 111.46 & 123.54 & 186.88 & 186.88 & 186.88 \\
\hline
\end{tabular}

Table 6 PRE of the proposed estimator $\tilde{\overline{\mathrm{Y}}}_{\mathrm{JS} 2}$ for the Population I

\begin{tabular}{|c|c|c|c|c|c|c|c|c|}
\hline \multirow[b]{2}{*}{$\alpha_{1}$} & \multirow[b]{2}{*}{$\alpha_{2}$} & \multirow{2}{*}{$\begin{array}{l}\text { SRSWOR } \\
\bar{y}_{\mathrm{r}}\end{array}$} & \multicolumn{6}{|c|}{ Existing estimators } \\
\hline & & & $\tilde{\overline{\mathrm{Y}}}_{1}$ & $\tilde{\overline{\mathrm{Y}}}_{2}$ & $\tilde{\overline{\mathrm{Y}}}_{3}$ & $\tilde{\overline{\mathrm{Y}}}_{4}$ & $\tilde{\overline{\mathrm{Y}}}_{5}$ & $\tilde{\overline{\mathrm{Y}}}_{6}$ \\
\hline 0 & 1 & 101.46 & 242.93 & 107.35 & 179.99 & 173.33 & 173.33 & 173.33 \\
\hline 0.1 & 0.9 & 102.14 & 244.56 & 108.07 & 167.94 & 161.54 & 161.54 & 161.54 \\
\hline 0.2 & 0.8 & 102.75 & 246.04 & 108.72 & 157.21 & 152.52 & 152.52 & 152.52 \\
\hline 0.3 & 0.7 & 103.31 & 247.36 & 109.31 & 147.84 & 146.36 & 146.36 & 146.36 \\
\hline 0.4 & 0.6 & 103.79 & 248.52 & 109.82 & 139.89 & 143.11 & 143.11 & 143.11 \\
\hline 0.5 & 0.5 & 104.21 & 249.51 & 110.26 & 133.38 & 142.83 & 142.83 & 142.83 \\
\hline 0.6 & 0.4 & 104.56 & 250.35 & 110.63 & 128.35 & 145.54 & 145.54 & 145.54 \\
\hline 0.7 & 0.3 & 104.84 & 251.03 & 110.93 & 124.82 & 151.29 & 151.29 & 151.29 \\
\hline 0.8 & 0.2 & 105.06 & 251.55 & 111.16 & 122.83 & 160.08 & 160.08 & 160.08 \\
\hline 0.9 & 0.1 & 105.21 & 251.92 & 111.32 & 122.39 & 171.93 & 171.93 & 171.93 \\
\hline I & 0 & 105.3 & 252.14 & 111.42 & 123.5 & $|86.8|$ & $|86.8|$ & $|86.8|$ \\
\hline
\end{tabular}

Table 7 PRE of the proposed estimator $\tilde{\overline{\mathrm{Y}}}_{\mathrm{JS} 1}$ for the Population I

\begin{tabular}{|c|c|c|c|c|c|c|c|c|}
\hline \multirow[b]{2}{*}{$\alpha_{1}$} & \multirow[b]{2}{*}{$\alpha_{2}$} & \multirow{2}{*}{$\begin{array}{l}\text { SRSWOR } \\
\bar{y}_{\mathbf{r}}\end{array}$} & \multicolumn{6}{|c|}{ Existing estimators } \\
\hline & & & $\tilde{\overline{\mathrm{Y}}}_{1}$ & $\tilde{\overline{\mathrm{Y}}}_{2}$ & $\tilde{\bar{Y}}_{3}$ & $\tilde{\overline{\mathrm{Y}}}_{4}$ & $\tilde{\overline{\mathrm{Y}}}_{5}$ & $\tilde{\overline{\mathrm{Y}}}_{6}$ \\
\hline 0 & I & 101.26 & 242.46 & 107.14 & 179.64 & 172.99 & 172.99 & 172.99 \\
\hline 0.1 & 0.9 & 101.97 & 244.17 & 107.9 & 167.67 & 161.28 & 161.28 & 161.28 \\
\hline 0.2 & 0.8 & 102.62 & 245.72 & 108.58 & $|57.0|$ & 152.32 & 152.33 & I52.33 \\
\hline
\end{tabular}


Table Continued...

\begin{tabular}{|c|c|c|c|c|c|c|c|c|}
\hline \multirow[b]{2}{*}{$\alpha_{1}$} & \multirow[b]{2}{*}{$\alpha_{2}$} & \multirow{2}{*}{$\begin{array}{l}\text { SRSWOR } \\
\bar{y}_{\mathbf{r}}\end{array}$} & \multicolumn{6}{|c|}{ Existing estimators } \\
\hline & & & $\tilde{\overline{\mathrm{Y}}}_{1}$ & $\tilde{\overline{\mathrm{Y}}}_{2}$ & $\tilde{\overline{\mathrm{Y}}}_{3}$ & $\tilde{\overline{\mathrm{Y}}}_{4}$ & $\tilde{\overline{\mathrm{Y}}}_{5}$ & $\tilde{\overline{\mathrm{Y}}}_{6}$ \\
\hline 0.3 & 0.7 & 103.2 & 247.11 & 109.2 & 147.7 & $|46.2|$ & 146.22 & 146.22 \\
\hline 0.4 & 0.6 & $103.7 \mid$ & 248.34 & 109.74 & 139.78 & 143.01 & 143.01 & 143.01 \\
\hline 0.5 & 0.5 & 104.16 & 249.4 & $\mid 10.21$ & 133.32 & 142.76 & 142.76 & 142.76 \\
\hline 0.6 & 0.4 & 104.54 & 250.31 & 110.61 & 128.32 & 145.52 & 145.52 & 145.52 \\
\hline 0.7 & 0.3 & 104.85 & 251.05 & 110.94 & 124.83 & 151.3 & 151.3 & 151.3 \\
\hline 0.8 & 0.2 & 105.09 & 251.64 & 111.2 & 122.87 & 160.13 & 160.13 & 160.14 \\
\hline 0.9 & 0.1 & 105.27 & 252.07 & 111.39 & 122.46 & 172.02 & 172.02 & 172.02 \\
\hline I & 0 & 105.39 & 252.34 & 111.51 & 123.6 & 186.97 & | 86.97 & | 86.97 \\
\hline
\end{tabular}

Table 8 PRE of the proposed estimator $\tilde{\bar{Y}}_{\text {JS4 }}$ for the Population I

\begin{tabular}{|c|c|c|c|c|c|c|c|c|}
\hline \multirow[b]{2}{*}{$\alpha_{1}$} & \multirow[b]{2}{*}{$\alpha_{2}$} & \multirow[t]{2}{*}{ SRSWOR } & \multicolumn{6}{|c|}{ Existing estimators } \\
\hline & & & $\tilde{\overline{\mathrm{Y}}}_{1}$ & $\tilde{\overline{\mathrm{Y}}}_{2}$ & $\tilde{\overline{\mathrm{Y}}}_{3}$ & $\tilde{\overline{\mathrm{Y}}}_{4}$ & $\tilde{\overline{\mathrm{Y}}}_{5}$ & $\tilde{\overline{\mathrm{Y}}}_{6}$ \\
\hline 0 & I & 101.47 & 242.95 & 107.36 & $|80.0|$ & 173.35 & 173.35 & 173.35 \\
\hline 0.1 & 0.9 & 102.15 & 244.59 & 108.08 & 167.96 & 161.55 & 161.55 & 161.55 \\
\hline 0.2 & 0.8 & 102.76 & 246.06 & 108.73 & 157.23 & 152.54 & 152.54 & 152.54 \\
\hline 0.3 & 0.7 & 103.31 & 247.38 & 109.32 & 147.86 & 146.37 & 146.37 & 146.38 \\
\hline 0.4 & 0.6 & 103.8 & 248.54 & 109.83 & 139.9 & 143.12 & 143.12 & 143.13 \\
\hline 0.5 & 0.5 & $104.2 \mid$ & 249.53 & 110.27 & 133.39 & 142.84 & 142.84 & 142.84 \\
\hline 0.6 & 0.4 & 104.56 & 250.37 & 110.64 & 128.35 & 145.55 & 145.55 & 145.55 \\
\hline 0.7 & 0.3 & 104.85 & 251.05 & 110.94 & 124.83 & 151.3 & 151.3 & 151.3 \\
\hline 0.8 & 0.2 & 105.06 & 251.57 & 111.17 & 122.84 & 160.09 & 160.09 & 160.09 \\
\hline 0.9 & 0.1 & 105.22 & 251.94 & 111.33 & 122.39 & 171.94 & 171.94 & 171.94 \\
\hline I & 0 & $|05.3|$ & 252.15 & 111.43 & 123.5 & 186.82 & 186.82 & 186.82 \\
\hline
\end{tabular}

Table 9 PRE of the proposed estimator $\tilde{\bar{Y}}_{\mathrm{JS} 1}$ for the Population 2

\begin{tabular}{|c|c|c|c|c|c|c|c|c|}
\hline \multirow[b]{2}{*}{$\alpha_{1}$} & \multirow[b]{2}{*}{$\alpha_{2}$} & \multirow{2}{*}{$\begin{array}{l}\text { SRSWOR } \\
\bar{y}_{\mathbf{r}}\end{array}$} & \multicolumn{6}{|c|}{ Existing estimators } \\
\hline & & & $\tilde{\overline{\mathrm{Y}}}_{1}$ & $\tilde{\overline{\mathrm{Y}}}_{2}$ & $\tilde{\overline{\mathrm{Y}}}_{3}$ & $\tilde{\overline{\mathrm{Y}}}_{4}$ & $\tilde{\overline{\mathrm{Y}}}_{5}$ & $\tilde{\overline{\mathrm{Y}}}_{6}$ \\
\hline 0 & I & 467.36 & 459.01 & 459.01 & 915.67 & 903.66 & 903.66 & 903.66 \\
\hline 0.1 & 0.9 & 619.38 & 608.3 & 608.3 & 1112.46 & 1092.73 & 1094.12 & 1094.81 \\
\hline 0.2 & 0.8 & 802.69 & 788.34 & 788.34 & $1326.0 \mid$ & $|3||.2|$ & 1314.35 & 1315.7 \\
\hline 0.3 & 0.7 & 1022.86 & 1004.57 & 1004.57 & $|56| .7 \mid$ & 1576 & 1581.14 & 1583.43 \\
\hline 0.4 & 0.6 & 1278.57 & $|255.7|$ & $|255.7|$ & $|8| 5.7 \mid$ & 1900 & 1907.86 & 1910.71 \\
\hline 0.5 & 0.5 & 1570.18 & 1542.11 & 1542.11 & 2092.11 & 2307.89 & 2316.67 & 2321.93 \\
\hline 0.6 & 0.4 & $\mid 864.58$ & 1831.25 & 1831.25 & 2356.25 & 2782.29 & 2790.63 & 2797.92 \\
\hline 0.7 & 0.3 & 2182.93 & 2143.9 & 2143.9 & 2648.78 & 3387.8 & 3393.9 & 3404.88 \\
\hline 0.8 & 0.2 & 2486.11 & 2441.67 & 2441.67 & 2938.89 & 4104.17 & 4104.17 & 4118.06 \\
\hline 0.9 & 0.1 & 2796.88 & 2746.88 & 2746.88 & 3271.88 & 4998.44 & 4990.63 & 5007.81 \\
\hline I & 0 & 3033.9 & 2979.66 & 2979.66 & 3567.8 & 5952.54 & 5932.2 & 5952.54 \\
\hline
\end{tabular}

Citation: Subramani J.Two parameter modified ratio estimators with two auxiliary variables for the estimation of finite population mean. Biom Biostat Int J. 20I8;7(6):559-568. DOI: 10.15406/bbij.2018.07.00259 
Table 10 PRE of the proposed estimator $\tilde{\overline{\mathrm{Y}}}_{\mathrm{JS} 2}$ for the Population 2

\begin{tabular}{|c|c|c|c|c|c|c|c|c|}
\hline \multirow[b]{2}{*}{$\alpha_{1}$} & \multirow[b]{2}{*}{$\alpha_{2}$} & \multirow{2}{*}{$\begin{array}{l}\text { SRSWOR } \\
\bar{y}_{\mathbf{r}}\end{array}$} & \multicolumn{6}{|c|}{ Existing estimators } \\
\hline & & & $\tilde{\overline{\mathrm{Y}}}_{1}$ & $\tilde{\overline{\mathrm{Y}}}_{2}$ & $\tilde{\overline{\mathrm{Y}}}_{3}$ & $\tilde{\overline{\mathrm{Y}}}_{4}$ & $\tilde{\overline{\mathrm{Y}}}_{5}$ & $\tilde{\overline{\mathrm{Y}}}_{6}$ \\
\hline 0 & 1 & 467.36 & 459.01 & 459.01 & 915.67 & 903.66 & 903.66 & 903.66 \\
\hline 0.1 & 0.9 & 619.38 & 608.3 & 608.3 & 1112.46 & 1092.73 & 1094.12 & 1094.81 \\
\hline 0.2 & 0.8 & 802.69 & 788.34 & 788.34 & $|326.0|$ & 13|| $.2 \mid$ & 1314.35 & 1315.7 \\
\hline 0.3 & 0.7 & 1011.3 & 993.22 & 993.22 & I544.07 & 1558.19 & 1563.28 & 1565.54 \\
\hline 0.4 & 0.6 & $|25| .75$ & 1229.37 & 1229.37 & 1777.62 & 1860.14 & 1867.83 & 1870.63 \\
\hline 0.5 & 0.5 & 1516.95 & 1489.83 & | 489.83 & 2021.19 & 2229.66 & 2238.14 & 2243.22 \\
\hline 0.6 & 0.4 & 1790 & 1758 & 1758 & 2262 & 2671 & 2679 & 2686 \\
\hline 0.7 & 0.3 & 2057.47 & 2020.69 & 2020.69 & 2496.55 & 3193.1 & 3198.85 & 3209.2 \\
\hline 0.8 & 0.2 & 2324.68 & 2283.12 & 2283.12 & 2748.05 & 3837.66 & 3837.66 & 3850.65 \\
\hline 0.9 & 0.1 & 467.36 & 459.01 & 459.01 & 915.67 & 903.66 & 903.66 & 903.66 \\
\hline I & 0 & 619.38 & 608.3 & 608.3 & 1112.46 & 1092.73 & 1094.12 & 1094.81 \\
\hline
\end{tabular}

Table II PRE of the proposed estimator $\tilde{\overline{\mathrm{Y}}}_{\mathrm{JS} 3}$ for the Population 2

\begin{tabular}{|c|c|c|c|c|c|c|c|c|}
\hline \multirow[b]{2}{*}{$\alpha_{1}$} & \multirow[b]{2}{*}{$\alpha_{2}$} & \multirow{2}{*}{$\begin{array}{l}\text { SRSWOR } \\
\bar{y}_{\mathbf{r}}\end{array}$} & \multicolumn{6}{|c|}{ Existing estimators } \\
\hline & & & $\tilde{\overline{\mathrm{Y}}}_{1}$ & $\tilde{\overline{\mathrm{Y}}}_{2}$ & $\tilde{\overline{\mathrm{Y}}}_{3}$ & $\tilde{\overline{\mathrm{Y}}}_{4}$ & $\tilde{\overline{\mathrm{Y}}}_{5}$ & $\tilde{\overline{\mathrm{Y}}}_{6}$ \\
\hline 0 & 1 & 464.94 & 456.62 & 456.62 & 910.91 & 898.96 & 898.96 & 898.96 \\
\hline 0.1 & 0.9 & 613.01 & 602.05 & 602.05 & 1101.03 & $1081.5 \mid$ & 1082.88 & 1083.56 \\
\hline 0.2 & 0.8 & 795.56 & 781.33 & 781.33 & $13 \mid 4.22$ & 1299.56 & 1302.67 & 1304 \\
\hline 0.3 & 0.7 & 1017.05 & 998.86 & 998.86 & 1552.84 & 1567.05 & 1572.16 & 1574.43 \\
\hline 0.4 & 0.6 & 1269.5 & $|246.8|$ & $|246.8|$ & 1802.84 & 1886.52 & 1894.33 & 1897.16 \\
\hline 0.5 & 0.5 & 1570.18 & I542.1I & 1542.11 & 2092.11 & 2307.89 & 2316.67 & 2321.93 \\
\hline 0.6 & 0.4 & 1904.26 & $|870.2|$ & 1870.21 & 2406.38 & 2841.49 & 2850 & 2857.45 \\
\hline 0.7 & 0.3 & 2237.5 & 2197.5 & 2197.5 & 2715 & 3472.5 & 3478.75 & 3490 \\
\hline 0.8 & 0.2 & 2594.2 & 2547.83 & 2547.83 & 3066.67 & 4282.61 & 4282.61 & 4297.1 \\
\hline 0.9 & 0.1 & 464.94 & 456.62 & 456.62 & 910.91 & 898.96 & 898.96 & 898.96 \\
\hline I & 0 & 613.01 & 602.05 & 602.05 & 1101.03 & $1081.5 \mid$ & 1082.88 & 1083.56 \\
\hline
\end{tabular}

Table 12 PRE of the proposed estimator $\tilde{\overline{\mathrm{Y}}}_{\mathrm{JS} 4}$ for the Population 2

\begin{tabular}{|c|c|c|c|c|c|c|c|c|}
\hline \multirow[b]{2}{*}{$\alpha_{1}$} & \multirow[b]{2}{*}{$\alpha_{2}$} & \multirow{2}{*}{$\begin{array}{l}\text { SRSWOR } \\
\bar{y}_{\mathbf{r}}\end{array}$} & \multicolumn{6}{|c|}{ Existing estimators } \\
\hline & & & $\tilde{\overline{\mathrm{Y}}}_{1}$ & $\tilde{\overline{\mathrm{Y}}}_{2}$ & $\tilde{\overline{\mathrm{Y}}}_{3}$ & $\tilde{\overline{\mathrm{Y}}}_{4}$ & $\tilde{\overline{\mathrm{Y}}}_{5}$ & $\tilde{\bar{Y}}_{6}$ \\
\hline 0 & 1 & 467.36 & 459.01 & 459.01 & 915.67 & 903.66 & 903.66 & 903.66 \\
\hline 0.1 & 0.9 & 615.12 & 604.12 & 604.12 & | I04.8I & 1085.22 & 1086.6 & 1087.29 \\
\hline 0.2 & 0.8 & 792.04 & 777.88 & 777.88 & $|308.4|$ & $|293.8|$ & 1296.9 & 1298.23 \\
\hline 0.3 & 0.7 & 1000 & 982.12 & 982.12 & 1526.82 & 1540.78 & $|545.8|$ & 1548.04 \\
\hline 0.4 & 0.6 & 1226.03 & |204.|I & 1204.11 & $|74| . \mid$ & 1821.92 & 1829.45 & 1832.19 \\
\hline 0.5 & 0.5 & 1479.34 & | 452.89 & 1452.89 & $197 \mid .07$ & 2174.38 & 2182.64 & 2187.6 \\
\hline 0.6 & 0.4 & 1737.86 & 1706.8 & 1706.8 & 2196.12 & 2593.2 & 2600.97 & 2607.77 \\
\hline
\end{tabular}


Table Continued...

\begin{tabular}{|c|c|c|c|c|c|c|c|c|}
\hline \multirow[b]{2}{*}{$\alpha_{1}$} & \multirow[b]{2}{*}{$\alpha_{2}$} & \multirow{2}{*}{$\begin{array}{l}\text { SRSWOR } \\
\bar{y}_{\mathbf{r}}\end{array}$} & \multicolumn{6}{|c|}{ Existing estimators } \\
\hline & & & $\tilde{\overline{\mathrm{Y}}}_{1}$ & $\tilde{\overline{\mathrm{Y}}}_{2}$ & $\tilde{\overline{\mathrm{Y}}}_{3}$ & $\tilde{\overline{\mathrm{Y}}}_{4}$ & $\tilde{\overline{\mathrm{Y}}}_{5}$ & $\tilde{\overline{\mathrm{Y}}}_{6}$ \\
\hline 0.7 & 0.3 & 1988.89 & 1953.33 & 1953.33 & 2413.33 & 3086.67 & 3092.22 & 3102.22 \\
\hline 0.8 & 0.2 & 2237.5 & 2197.5 & 2197.5 & 2645 & 3693.75 & 3693.75 & 3706.25 \\
\hline 0.9 & 0.1 & 467.36 & 459.01 & 459.01 & 915.67 & 903.66 & 903.66 & 903.66 \\
\hline I & 0 & 615.12 & 604.12 & 604.12 & | | $04.8 \mid$ & 1085.22 & 1086.6 & 1087.29 \\
\hline
\end{tabular}

\section{Concluding remarks}

In this paper, two parameter modified ratio estimators using the linear combination of the correlation coefficient and skewness of auxiliary variables has been suggested. The mean squared error of the proposed estimators are derived and compared with that of the SRSWOR sample mean, the classical ratio estimator and the existing modified ratio estimators. Further we have derived the conditions for which the proposed estimators are more efficient than the existing estimators. We have also assessed the performance of the proposed estimators with that of the existing estimators for certain natural populations. It is observed that the mean squared error of the proposed estimators is less than the mean squared error of the existing estimators for two populations. Further it has been shown that the efficiency of the proposed estimators with respect to existing estimators are in general ranging from 101.26 to 5007.81. Hence we strongly recommend that the proposed modified ratio estimators may be preferred over the existing estimators for practical applications.

\section{Acknowledgement}

None.

\section{Conflict of interest}

Author declares that there is no conflict of interest.

\section{References}

1. Cochran WG. Sampling Techniques. New York: John Wiley \& Sons; 1977.

2. Murthy MN. Sampling Theory and Methods. Calcutta: Statistical Publishing Society; 1967.

3. Sisodia BVS, Dwivedi VK. A modified ratio estimator using coefficient of variation of auxiliary variable. Journal of the Indian Society of Agricultural Statistics. 1981;33(1):13-18.

4. Singh HP, Tailor R, Kakran MS. Improved estimators of population mean using power transformation. Journal of the Indian Society of Agricultural Statistics. 2004;58(2):223-230.

5. Upadhyaya LN, Singh HP. Use of transformed auxiliary variable in estimating the finite population mean. Biometrical Journal. 1999;41(5):627-636.

6. Subramani J, Kumarapandiyan G. Estimation of population mean using known median and co-efficient of skewness. American Journal of Mathematics and Statistics. 2012;2(5):101-107.

7. Subramani J, Kumarapandiyan G. Estimation of population mean using co-efficient of variation and median of an auxiliary variable. International Journal of Probability and Statistics. 2012;1(4):111-118.

8. Subramani J, Kumarapandiyan G. Modified ratio estimators using known median and co-efficient of kurtosis. American Journal of Mathematics and Statistics. 2012;2(4):95-100.
9. Subramani J, Kumarapandiyan G. A new modified ratio estimator for estimation of population mean when median of the auxiliary variable is known. Pakistan Journal of Statistics and Operation Research. 2013;9(2):137-145.

10. Abu-Dayyeh WA, Ahmed MS, Ahmed RA, et al. Some estimators of finite population mean using auxiliary information. Applied Mathematics and Computation. 2003;139:287-298.

11. Bandyopadhyay S. Improved ratio and product estimators. Sankhyā C. 1980;42:45-49.

12. Kadilar C, Cingi H. Estimator of a population mean using two auxiliary variables in simple random sampling. International Mathematical Journal. 2004;5:357-360.

13. Kadilar C, Cingi H. A new estimator using two auxiliary variables. Applied Mathematics and Computation. 2005;162:901-908.

14. Khare BB, Srivastava U, Kumar K. A generalized chain ratio in regression estimator for population mean using two auxiliary characters in sample survey. Journal of Scientific Research. 2013;57:147-153.

15. Naik VD, Gupta PC. A general class of estimators for estimating population mean using auxiliary information. Metrika. 1991;38:11-17.

16. Olkin I. Multivariate ratio estimation for finite populations. Biometrika. 1958;45:154-165.

17. Perri PF. Alcune considerazioni sull'efficienza degli stimatori rapportocum-prodotto. Statistica \& Applicazioni. 2004;2(2):59-75.

18. Perri PF. Improved ratio-cum-product type estimators. Statistics in Transition-NS. 2007;8(1):51-69.

19. Rao PSRS, Mudholkar GS. Generalized multivariate estimator for the mean of finite populations. Journal of the American Statistical Association. 1967;62:1009-1012.

20. Raj D. On a method of using multi-auxiliary information in sample surveys. Journal of the American Statistical Association. 1965;60:154 165.

21. Sahoo LN, Swain AKPC. Unbiased ratio-cum-product estimator Sankhyā C. 1980;42:56-62.

22. Singh MP. On the estimation of ratio and product of the population parameters. Sankhyā B. 1965;27:321-328.

23. Singh MP. Multivariate product method of estimation for finite populations. Journal of the Indian Society of Agricultural Statistics. 1967;31:375-378.

24. Singh MP. Ratio cum product method of estimation. Metrika. $1967 ; 12: 34-42$.

25. Srivenkataramana T. A dual to ratio estimator in sample surveys Biometrika. 1980;67:199-204.

26. Srivenkataramana T, Tracy DS. An alternative to ratio method in sample surveys. Annals of the Institute of Statistical Mathematics. 1981;32:111120 . 
27. Tailor R, Parmar R, Kim JM, et al. Ratio-cum-Product estimators of population mean using known population parameters of auxiliary variable. Communication of the Korean Statistical Society. 2011;18(2):155-164.

28. Tracy DS, Singh HP, Singh R. An alternative to the ratio-cum-product estimator in sample surveys. Journal of Statistical Planning and Inference. 1996;53:375-387.

29. Singh D, Chaudhary FS. Theory and analysis of sample survey designs. New Age International Publisher. 1986.

30. Kadilar C, Cingi H. Advances in Sampling Theory- Ratio Method of Estimation. Bentham Science Publishers. 2009.

31. Singh GN. On the improvement of product method of estimation in sample surveys. Journal of the Indian Society of Agricultural Statistics. 2003;56(3):267-265.
32. Singh HP, Tailor R. Estimation of finite population mean using known correlation coefficient between auxiliary characters. Statistica. 2005;65:407-418.

33. Singh HP, Tailor R. Use of known correlation coefficient in estimating the finite population means. Statistics in Transition. 2003;6(4):555-560.

34. Cochran WG. The estimation of the yields of cereal experiments by sampling for the ratio of grain to total produce. Journal of Agriculture Science. 1940;37:199-212.

35. Yan Z, Tian B. Ratio method to the mean estimation using coefficient of skewness of auxiliary variable, ICICA 2010, Part II, CCIS 106. 2010;103-110. 